\title{
Geriatrics Evaluation and Management in the Veterans Administration- An Historical Perspective
}

\author{
James S. Powers ${ }^{1}$ \\ Kathryn J. Eubank ${ }^{2}$
}

From Vanderbilt University School of Medicine, and the Tennessee Valley Healthcare System Geriatrics Research, Education, and Clinical Center, Nashville TN ${ }^{1}$; The University of California San Francisco School of Medicine and the San Francisco VA Medical Center, San Francisco, $C A^{2}$

\section{Corresponding Author}

James S. Powers MD

Clinical Associate Director, Tennessee Valley Healthcare System

Geriatric Research Education and Clinical Center

7159 Vanderbilt Medical Center East

Nashville TN 37232

615-936-3274

FAX 615-936-3156

James.powers@vanderbilt.edu 


\begin{abstract}
Comprehensive geriatric assessment, defined as an interdisciplinary assessment and development of an overall plan of treatment and follow-up, has become a fundamental part of clinical geriatric care. Since the 1970s, the Veterans Administration has encouraged the development of geriatric evaluation and management programs. Evolution of geriatric evaluation and management has occurred over time and many VA medical centers have transferred inpatient geriatric evaluation programs to long-term care Community Living Centers (CLC) and outpatient settings. Availability of resources and trained personnel across the continuum of care as well as administrative facilitation of collaboration between care components are critical to the efficient utilization of geriatric services. Facilities may need to prioritize their resources and utilize the most effective and relevant elements of geriatric evaluation and management according to patient population needs, available space, resources, and institutional priorities.
\end{abstract}

Keywords: Geriatric Evaluation and Management, Acute Care for Elders, Geriatric Rehabilitation 


\section{Introduction}

Comprehensive geriatric assessment, defined as an interdisciplinary assessment and development of an overall plan of treatment and follow-up, has become a fundamental part of clinical geriatric care. Since the 1970s, the Veterans Administration has encouraged the development of Geriatric Evaluation and Management (GEM) programs, with at least 105 inpatient GEM programs reported in $1993 .{ }^{1}$ Standard GEM programs consist of 1) interdisciplinary core team composition including a physician, nurse, and social worker (with additional personnel as appropriate for individual patients i.e. dietitian, therapists, pharmacist, psychologist), 2) comprehensive assessment, 3 ) selective admissions for patients with potential to benefit from GEM, 4) location in acute or long term care location, and 5) provision for inpatient rehabilitation and follow-up. ${ }^{1}$ Nonstandard units lack one or more of these characteristics. Inpatient geriatric units are associated with improved diagnostic accuracy and management of geriatric syndromes, reduced numbers of medications, improved functional outcome, fewer long-term care admissions, and reduced acute care hospital readmissions, improved medication utilization, and establishment of patient-centered goals of care. ${ }^{2-4}$ A 1993 VA GEM program assessment revealed the average GEM unit then consisted of a mean 12.7 beds (standard unit) to 15.4 beds (non-standard unit) size, with a mean length of stay (LOS) 24.5 days (standard unit) to 69.9 days (non-standard unit). ${ }^{1}$ Since that time other geriatric evaluation models have been developed. Acute Care for Elders (ACE) Units are specially designed units located in the acute care setting and focused on maintaining function, early discharge planning, and patient-centered ageappropriate care. ${ }^{2,4}$ A meta-analysis of ACE programs published in 2012 showed that ACE Units, compared to usual hospital care, had significantly lower length of stays, lower hospital costs, and more discharges home. In addition, the ACE model was associated with fewer falls, less delirium, and less functional decline. ${ }^{5}$ Mobile ACE Units (MACE), acute care geriatric services without a geographic bed location, have since been shown to have similar outcomes. ${ }^{6}$

The VA requires availability of geriatric evaluation services for all military veterans as mandated in the 1999 Veterans Millennium Healthcare and Benefits Act (Mill Bill), and these services may currently be located in inpatient, long-term care - community living center (CLC), or outpatient settings. ${ }^{7}$ Geriatric evaluation consists of a comprehensive, multi-dimensional assessment and development of an interdisciplinary plan of care undertaken by an interdisciplinary team of healthcare professionals for a target a group of high-risk high-need patients who are most likely to benefit from these services. Over time many VA medical centers have transferred inpatient geriatric evaluation programs to long-term care and outpatient settings. We track the evolution of GEM services at Tennessee Valley Healthcare System (TVHS), which transferred its Geriatric Evaluation Unit to the CLC in November 2017, and contrast the TVHS experience with GEM to the ACE Unit at the San Francisco VA Medical Center (SFVAMC), discussing geriatric evaluation services in the VA from a 30-year clinical, operations and policy perspective. 


\section{Methods}

\section{Setting}

A GEM Unit was initiated in 1987 at the Nashville Campus of TVHS $^{8}$ as an intermediate care (IMC) unit, a VA geographic bed designation with an average length of stay less than 30 days, from 1987-2000. The focus included geriatric rehabilitation and preserving function. The size of the unit averaged 10 beds during that time with an average of 2 to 4 geriatric consults per day. Approximately $50 \%$ of consults resulted in a transfer to the unit, with the remainder managed with recommendations to reduce risk of hospital acquired conditions, management of geriatric syndromes, and disposition recommendations. In 2000, TVHS united the Nashville and 40-mile distant Murfreesboro Campuses under one administration. In that year the IMC designation was withdrawn due to acute care space constraints at the Nashville Campus and the unit functioned as an ACE unit without contiguous bed designation. The VA Nashville campus operated 393 acute care beds in $1991,{ }^{8}$ and facility utilization changed over time with development of outpatient services and a gradual reduction of the acute care service to 238 beds (40\% reduction) by 2017. In November 2017 the GEM Unit was transferred to the CLC as a four-bed unit at the Murfreesboro Campus. The GEM Unit performed over 12,000 consults and cared for a total of 5380 patients at the Nashville campus over a 30-year time frame.

The ACE unit was initiated at the San Francisco VA Medical Center in 2010 occupying 10-beds of a 20-bed geographically-based acute care specialty unit, focused on preventing iatrogenic complications in high-risk, high-need community dwelling adults 65 years of age and older admitted to the hospital for an acute illness. The unit included traditional elder-friendly elements of an ACE unit, such as uncluttered hallways, handrails, large clocks, low beds, elevated toilet seats, and large writing boards to post information. Also included was a community room where Veterans could interact with staff or other patients, eat meals together, and participate in activities that enhance mobility and cognitive abilities. The ACE service was comprised of a full-time geriatrician (MD), a full time geriatric certified nurse specialist (CNS), and shared time physical therapist (PT), occupational therapist (OT), social worker, and pharmacist. The service initially operated as a geographically contained unit with automatic consultation for patients aged 65 years of age and older admitted to the unit. The primary team managed the acute illness and the ACE team focused on preserving function, preventing or treating delirium, and addressing polypharmacy and medication management during the hospitalization. The ACE Service experienced high demand for services and in order to better meet the needs of older adults across the medical center, in 2017 it transitioned to a mobile ACE (MACE) model screening all veterans admitted to the medical service age 85 years and older, regardless of location. The geriatric consult focused on management of geriatric syndromes, avoidable acute care complications, with optional transfer to the MACE Service. SFVAMC has a capacity of 124 acute care beds with a closely affiliated adjacent 120-bed CLC.

\section{Data Collection}


TVHS GEM operations data was collected from 1987 to 2017 and included age, length of stay, disease state, disposition, and mortality data. Run chart analysis of trends was performed with descriptive and comparative statistics applied. The SFVAMC collected length of stay, readmission rates, average daily census, and discharge to long-term-care between 2011-2016. Since 2017, the MACE program has collected age and reason for consult request data for the geriatric consult service. Historical program development information from both sites was provided by the contributing authors.

The TVHS-Institutional Review Board has designated this project as Quality Improvement.

\section{Results}

The TVHS GEM Unit had a mean patient age of 73(2.4) years, and the proportion of patients over age 65 averaged $69(8.2) \%$. While approximately $30 \%$ of patients with anticipated discharge to long-term care were able to return home, there was a progressive decline in the percentage of those over age 65 with time $\left[\mathrm{r}-.75\left(\mathrm{r}^{2} .58\right), \mathrm{p}<.0001\right]$ and a corresponding increase in the medically complex population $\left[\mathrm{r} .67\left(\mathrm{r}^{2} .45\right), \mathrm{p}<.0001\right]$. The 30 -day readmission rate remained $10 \%$ between $2015-17$.

GEM functioned predominantly as a 10-bed unit, but size varied between 6-26 beds at different points depending on space availability and facility need. There were increased transfers to longterm care, especially after 2005 [F=49, p<.001], (Figure 1) with mean 27(13)\% discharges to long-term care sent to the Murfreesboro CLC by 2015-2017. Additionally there were increased numbers of transfers to the Murfreesboro Hospice Unit following its creation in 2000, with 38\% Nashville patients occupying the 12 bed unit in 2016-17. (Table 1)

GEM mean length of stay (LOS) dropped dramatically from the 1990s when LOS was 29.9(4.8) days to $16.2(1.3)$ days from $2000-2017$ [F=11.76, $\mathrm{p}<.01]$. However, the length of stay was unchanged from 2000-2017, reflecting a constant average 16 days for these high-risk, high-need patients. The GEM population included geriatric rehabilitation and at times also homeless patients, transplant patients, dementia patients and other difficult-to-place patients depending on facility demands.

\section{Table 1. GEM Utilization Trends (TVHS 1987 - 2017)}

- decline in population over age 65

- increase in the medically complex population

- $\quad$ Length of stay unchanged from 2000 to 2017 
- increased transfers to Murfreesboro CLC

- increased transfers to Murfreesboro Hospice

\section{Figure 1. Discharge to LTC (\%)}

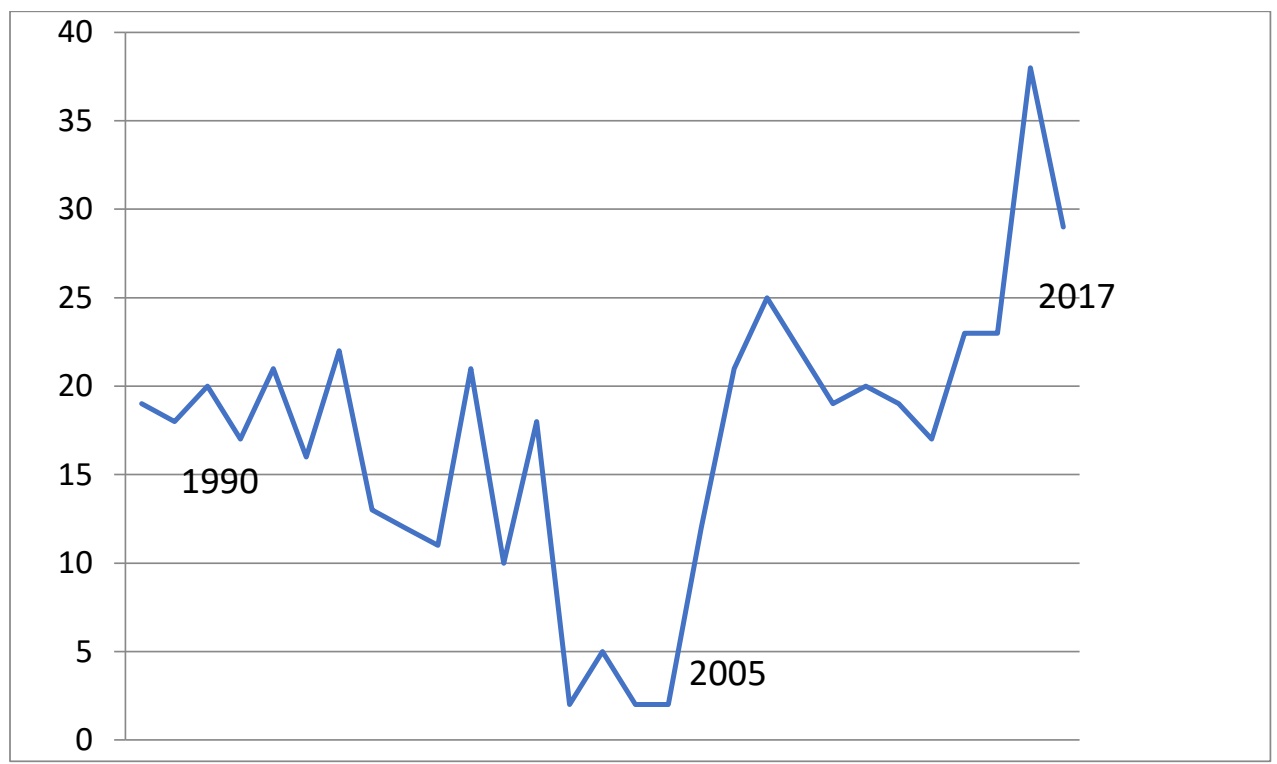

Mean \% Discharge to LTC (1987-2005) 13.5(3.6)\%, (2006-2017) 22.3(4.1)\%, F=11.76, p<.01 
Figure 2. Post Gem Transfer LOS (days)

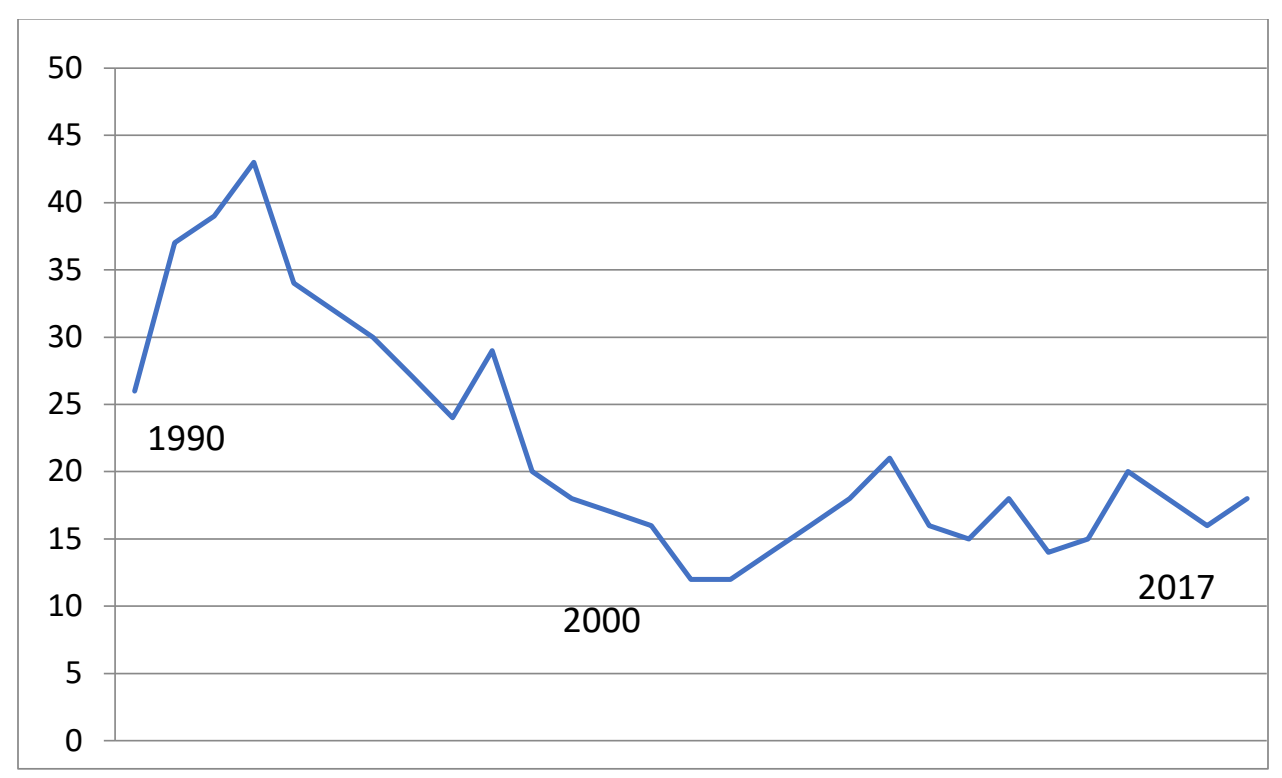

Mean LOS (1987-2000) 29.9(4.8) days, (2001-2017) 16.2(1.3) days, F=49, p<.0001

Major changes at TVHS over the development timeline for GEM are listed in Table 2. This included the development of primary care and outpatient services during the 1990s and a case manager program to improve patient-centered care and to facilitate discharge planning. In 1995 a Geriatric Psychiatry and in 1999 a Geriatric Medicine Fellowship program and a Geriatric Research, Education, and Clinical Center (GRECC) were established. These developments led to enhanced geriatric training for all healthcare disciplines, eventually recruiting an additional 8 trained geriatricians and many advanced practice nurses, clinical pharmacists, psychologists, and social workers to the clinical staff. This increase in clinical expertise permitted the development of a hospice care unit in Murfreesboro in 2000, and enhanced the ability to accept and care for increased patient acuity in the CLC. The 2000 unification of the Nashville and Murfreesboro Campuses gradually improved coordination and increased the availability of long-term care services. Competing acute care space constraints increased at the Nashville Campus with the introduction of specialized heart failure, and bone marrow, liver, and cardiac transplant services.

Geri-PACT $^{9}$ was initiated in 2011 to provide enhanced outpatient geriatric evaluation, becoming widespread with over 60 Geri-PACT programs operating nationally by 2014. In 2016 the MidSouth Healthcare System, which includes TVHS, mandated Geri-PACT at all facilities as part of its strategic plan. In 2015 the Strategic Analytics for Improvement and Learning Value Model (SAIL) was introduced by the Department of Veterans Affairs to measure, evaluate, and benchmark quality and efficiency internally at its medical centers. SAIL is a web-based, balanced scorecard focused on value-based population management with 9 domains: Performance Measures, Patient Satisfaction, Mortality, Length of Stay, Access, Efficiency, 
Ambulatory Care Sensitive Conditions, Hospitalizations, and Avoidable Adverse Events. ${ }^{10}$ The advent of SAIL and a publicly reported 5-Star rating system in 2016 heavily influenced administrative performance objectives regarding quality indicators and customer satisfaction. TVHS received an initial 1-Star rating in 2016, and advanced to a 2-Star rating in 2018. In the $4^{\text {th }}$ quarter of 2017, the TVHS CLC was awarded the highest 5-Star rating.

During its years of operation, the SFVAMC Ace Unit ran an average daily census of 13 (6-18) veterans aged 65 and older. The LOS steadily decreased over the period of operation, from 7.9 days in 2011 to 5.3 days in 2016, consistent with national acute care trends. The 30-day readmission rate to the SFVAMC for those served on the ACE Unit was $4.1 \%$ (national VA average $18.4 \%$ in 2012) with a discharge to a skilled nursing facility (SNF) of $39.5 \%$ (national VA average in 2012 was 27\%). By design, the SFVAMC ACE model specifically focused on preserving/regaining function, preventing/treating delirium, and medication review. Other geriatric syndromes or care needs were addressed as needed. The ACE Unit was a primary training environment for geriatric fellows ( 2 required rotations/year) as well as elective rotations for medical students, internal medicine residents, advanced practice nursing students, pharmacy residents and students, and therapy students. Despite efforts to triage patients appropriately between the ACE Unit and other general patient units, due to the small hospital size and bedavailability, it was not unusual that the geographic ACE Unit would have patients admitted in less need of our services, for example meeting age but not functional criteria, while older or more frail patients who would benefit from geriatric consultation would be admitted to another unit. After initiating the MACE model, automatic screening of all adults aged 85 and older was performed as close to admission as possible. The geriatrics consult service averaged $3.2(0-7)$ new formal geriatrics consults/week with an average age of 78 (62-91). The most common reasons for consultation were delirium $39 \%$,failure to thrive $18 \%$, treatment of dementia related behaviors $15 \%$, assistance with polypharmacy or medication management $10 \%$, and falls $10 \%$. Other areas included capacity assessment, peri-operative management, help with dispositions, elder abuse, depression, and urinary retention. Many consults included requests for management of several conditions.

Like TVHS, the SFVAMC has HBPC, Geri-PACT clinic as well as outpatient geriatrics consult and telehealth services. In addition, the SFVAMC has a high-needs, intensive outpatient management consult team called IMPACT (intensive management patient aligned care team) that works with PACT providers (geri and non-geri) to assist patients in times of high medical or social need. The San Francisco VA Medical Center has a 3-Star designation, and has a limited CLC capacity. 


\section{Table 2. Program Milestones}

TVHS Milestones since 2000

- Development of primary care and outpatient services $1990 \mathrm{~s}$

- Development of a case manager program

- 1990's development of Geriatric Fellowship programs to train faculty

- 1999 TVHs GRECC awarded

- 2000 unification of campuses under one administration

- 2000 development of a hospice care unit, Murfreesboro Campus

- Increase in transplant population and specialty services

- Increased availability of CLC since consolidation

- Competing acute care space constraints, geographic IMC bed designation cessation

- Geri-PACT initiated 2011, VISN mandated 2016

- 2015 SAIL Metrics and 5-Star rating system

San Francisco ACE Milestones

- $\quad$ Strong Geriatric training program since 1997

- 2010, shared 20-bed geographic ACE Unit

- 2017 MACE service without geographic bed designation, seeing all patients $>$ age 85

- 2017 Inpatient geriatrics consult screening for all medical patients over age 85

- Small CLC capacity and bed availability

- Recognition of ACE educational value and contribution to SAIL Metrics and 5-Star rating system

\section{Discussion}

Both services are located in busy acute-care hospitals with active geriatric training programs and have a similar consultation rate of 2 to 4 patients per day, but with different foci. The SFVAMC provides mobile acute-care for elder patients focusing on older and frailer adults most likely to benefit from interventions, with additional consult services to younger adults as deemed needed by the primary teams. Once discharged from the acute setting, the MACE team does not follow the patients into the CLC or other SNFs for rehab or subacute care. However, TVHS also targeted potential rehabilitation and difficult-to-place patients, rehabilitating some $30 \%$ to achieve home discharge. The TVHS GEM program experienced a gradual increase in care for complex medical patients and a decline in mean age concurrent with an increase in medical specialty and transplant program expansion at the Nashville Campus. The benefits of GEM included a reduced 30-day acute-care readmission, improved functional status, reduced numbers of medications, and improved continuity of care for complex patients requiring specialty 
consultation. GEM improved the ability to address social and behavioral determinants of health and to provide continuity of inpatient care with a patient-centric discharge planning process for high-risk, high-need patients. The service also off-loaded teaching services, provided a stable interdisciplinary treatment team with consistency of staffing and program focus. It also facilitated geriatric education and research, and created a model interdisciplinary team. ${ }^{8}$ However the cost of GEM included interdisciplinary team personnel with required time commitments competing with other facility program needs, as well as competition for acute care beds. A 16-day GEM length of stay persisted since 2000, despite the unification of campuses. The opportunity cost for GEM included the availability of alternative care sites capable of complex care, especially CLC and Hospice at the Murfreesboro Campus, staffed by providers trained by the facility's Geriatric Medicine and Psychiatry programs.

Priorities favoring geriatric evaluation location in acute care include a focus in preventing acute care iatrogenic complications, medical center leadership viewing it as a priority helpful in achieving SAIL metrics, valuing the educational role of the unit, and CLC availability. While loss of a contiguous bed designation could produce challenges to maintain the geriatric environment of care (Table 3) due to lack of consistent dedicated unit staffing, it provides greater opportunity for education of staff across units resulting in "geriatricizing" non-geriatrics clinicians, including physicians, nurses, and aides. For example, it is not unusual now that the instigator of a consult may be a nurse on the intensive care step down unit, noticing mental status changes in a patient and implementing delirium precautions on their own while asking physicians to call a geriatrics consult for assistance.

\section{Table 3. Inpatient Geriatric Evaluation Beds}

\section{Benefits}

- Reduced 30-day acute care readmission

- Continuity of care for complex patients requiring specialty consultation

- Stable interdisciplinary team, and consistency of staffing

- Ability to address social behavioral determinants of health

- Facilitation of geriatric education and research

\section{Costs}

- Interdisciplinary team member cost, availability

- Acute care space at a premium

- Availability of alternative care sites and dispositions

- Persistently prolonged length of stay for difficult-to-place patients 


\section{Priorities Favoring Geriatric Evaluation in Acute Care}

- Acute care focus on preventing iatrogenic complications, maintaining function

- Medical Center leadership views as a priority

- Hospital size, length of stay, space availability

- Educational mission

- Contribution to achieving SAIL metrics

\section{Priorities Favoring Geriatric Evaluation in CLC and Outpatient Locations}

- Availability of space and resources in CLC, Outpatient

- Availability of trained staff in CLC, Outpatient for high-risk high-need patients

- Administrative commitment to close cooperation among resource units

- Rehabilitation focus

Outpatient geriatric evaluation is available through primary care Geriatric Patient Aligned Care Teams (Geri-PACT) a patient-centered medical home which provides care for frail elderly Veterans by a geriatrics-trained interdisciplinary team, where more complex patients with involved medical histories can receive in-depth attention. Care is provided to geriatric patients through coordinated, interdisciplinary provision of medical, nursing, psychosocial, allied health services for disease treatment and prevention, health promotion and education, referral for specialty, rehabilitation and other levels of care, follow-up and overall care management by the primary care provider and support team. ${ }^{9}$

Consultation Geriatrics may be another efficient means to employ scarce geriatric resources. The effectiveness of consult care could be diminished due to the limited ability to control interventions, however greater availability of patient-centered primary care at the VA (Primary Care PACT) accepting ownership of patient management across the continuum of care, combined with efficient utilization of home and community based resources may be an effective strategy to enhance geriatric evaluation and care for patients not followed in Geri-PACT.

Home Based Primary Care (HBPC) includes health care services provided to Veterans in their home. A VA physician supervises the health care team providing the services. Home Based Primary Care is for Veterans who have complex health care needs for whom routine clinic-based care is not effective, or for whom travel for office visits is not possible. ${ }^{11}$

A review of program elements comprising ACE Units identified three key concepts: 1) geriatric medical review, 2) early rehabilitation and 3) patient-centered care. These individual, focused interventions appear to have a greater effect than either discharge planning processes, or environment of care. ${ }^{2}$ Since its inception in the 1970s, there has been an evolution of VA 
geriatric evaluation and management over time and resources have become more available in long-term care and outpatient settings. VA Geriatric evaluation services now comprise 34 Inpatient (1 mobile ACE and remainder CLC programs), 64 Outpatient GERI-PACT, ${ }^{12}$ over 116 HBPC, ${ }^{11}$ and many skilled and non-skilled home and community-based services as result of the Mill Bill.

The marked variability in the reported size and length of stay of different geriatric evaluation programs suggests that program location may be influenced by local needs and availability of workforce and resources. Previous reviews of geriatric evaluation availability and program closure or relocation suggest that the availability of interdisciplinary team members and sufficient resources for geriatric evaluation services are critical. ${ }^{1}$ Our experience suggests that patient population characteristics, length of stay, and availability of trained personnel and alternative placements capable of meeting the needs of high-risk, high-need patients may also be important considerations. The VA guidelines appear to be adapting to evolution of the model, including the addition of outpatient and in-home evaluation as alternative sites for geriatric evaluation programs.

Patient selection consistent with facility priorities is important to ensure that geriatric evaluation services are placed in the appropriate location and provided to appropriate patient populations. Hospital size, length of stay, and competing space obligations are important local factors to consider. Availability of resources and trained personnel across the continuum of care as well as administrative facilitation of collaboration between care components are critical to the efficient utilization of geriatric evaluation services.

\section{Future Direction and Policy Implication}

Barriers exist to maintaining acute care geriatric models, as acute care beds are at a premium in most medical centers. Facilities may need to prioritize their resources and utilize the most effective and relevant elements of geriatric evaluation and management according to the patient population, available space, resources, and institutional priorities. 


\section{References}

1.Wieland D, Rubenstein LZ, Hedrick SC, Reuben DB, Buchner DM. Inpatient geriatric evaluation and management units (GEMs) in the veterans health system: diamonds in the rough? J Gerontol. 1994;49:195-200.

2. Fox MT, Sidani S, Persaud M, Tregunno D, Maimets I, Brooks D, O’Brien K. Acute care for elders components of acute geriatric unit care: Systematic descriptive review. JAGS 2013;61: 939-946.

3. Reubenstein LZ, Josephson KR, Weiland GD, English PA, Sayre JA, 1Kane RL.

Effectiveness of a geriatric evaluation unit. A randomized clinical trial. NEJM 1984;311:16641670.

4. Landefeld CS, Palmer RM, Kresevec DM, Fortinsky RH, Kowal J. a randomized trial of care hospital medical unit especially designed to improve the functional outcomes acutely ill older patients. NEJM 1995; 332:1338 - 1334.

5. Fox MT, Persaud M, Maimets I et al. Effectiveness of acute geriatric unit care using Acute Care for Elders components: A systematic review and meta-analysis. J Am Geriatr Soc 2012;60:2237-2245.

6. Hung WW, Ross JS, Farber J, Siu AL. Evaluation of the Mobile Acute Care of the Elderly (MACE) service. JAMA internal medicine. 2013;173(11):990-996.

7. Department of Veterans Affairs, VHA Directive 1140.04. Geriatric Evaluation. November 28, 2017. https://www.va.gov/vhapublications/ViewPublication.asp?pub_ID=5619 (Accessed July 10, 2018)

8. Hauser B, Robinson J, Powers JS, Laubacher MA. The evaluation of an intermediate caregeriatric evaluation unit in a veterans administration Hospital. Southern Medical Journal. 1991;84:597-602.

9. Department of Veterans Affairs. Geri-PACT Handbook 1101.10 (1). www.va.gov/vhapublications/ViewPublication.asp?pub_ID=2977 (Accessed July 10, 2018)

10. VA Sail Metrics http://www.va.gov/QUALITYOFCARE/measure-up/SAIL_definitions.asp (Accessed July 10, 2018)

11. Beales JL, Edes T. Veteran's Affairs Home Based Primary Care. Clin Geriatr Med. 2009;25:149-54.

12. Department of Veterans Affairs. Patient Care Services. Geriatrics and Extended Care Program. https://www.patientcare.va.gov/geriatrics.asp (Accessed July 10, 2018) 